\title{
Le symbolisme alchimique de "The Black Tower », dernier poème de Yeats
}

\section{Michel Dufour}

\section{(2) OpenEdition \\ 12 Journals}

\section{Édition électronique}

URL : http://journals.openedition.org/etudesirlandaises/2427

DOI : 10.4000/etudesirlandaises. 2427

ISSN : 2259-8863

\section{Éditeur}

Presses universitaires de Rennes

\section{Édition imprimée}

Date de publication : 30 décembre 2011

Pagination : 119-128

ISSN : 0183-973X

\section{Référence électronique}

Michel Dufour, "Le symbolisme alchimique de «The Black Tower », dernier poème de Yeats », Études irlandaises [En ligne], 36-2 | 2011, mis en ligne le 30 septembre 2013, consulté le 02 mai 2019. URL http://journals.openedition.org/etudesirlandaises/2427 ; DOI : 10.4000/etudesirlandaises.2427

Ce document a été généré automatiquement le 2 mai 2019.

(c) Presses universitaires de Rennes 


\title{
Le symbolisme alchimique de « The Black Tower ", dernier poème de Yeats
}

\author{
Michel Dufour
}

1 Il s'agit du dernier poème que W. B. Yeats écrivit, le 21 janvier 1939, exactement une semaine avant sa mort. Le matin du 28 janvier 1939, Yeats serait sorti de son coma et aurait encore apporté quelques corrections au poème ${ }^{1}$, avant de mourir, à 14 heures, à Roquebrune-Cap-Martin. S'il est intéressant de saisir les derniers instants poétiques d'un des plus grands poètes du $\mathrm{xx}^{\mathrm{e}}$ siècle et de considérer l'éclairage qu'ils peuvent porter sur l'ensemble de l'œuvre, l'entreprise revêt d'autant plus d'importance dans le cas de Yeats que celui-ci avait pour préoccupation première l'unité de son œuvre et qu'il retravaillait sans cesse tous ses poèmes pour présenter une œuvre poétique achevée et unifiée. A ses amis qui s'interrogeaient sur son besoin constant de corriger et recorriger ses anciens poèmes, Yeats répondait : «It is myself that I remake ${ }^{2}$. " Et à propos des mêmes révisions il déclarait: «One is always cutting out the dead wood ${ }^{3}$.» Cette préoccupation trouvait sa justification théorique dans le concept d'Unité d'Être que Yeats définit à partir de 1920 et qui établit comme loi de l'esthétique que dans leurs interrelations, les multiples éléments d'une œuvre doivent chacun contribuer à l'harmonie du tout. Les derniers poèmes traduisent la recherche d'un point final clôturant l'œuvre d'une manière définitive, selon différents points de vue. «The Man and the Echo » pose le problème de la responsabilité morale du poète dont l'œuvre a influencé le destin personnel de ses amis, mais aussi l'histoire tragique de son pays : «Did that play of mine send out / Certain men the English shot ${ }^{4}$ ?» «Under Ben Bulben » se présente comme le testament poétique officiel où le poète, parlant d'outre-tombe, enjoint à ses successeurs de suivre un programme thématique et esthétique particulier: "Irish poets learn your trade / Sing whatever is well made [... $]^{5}$.» «Cuchulain Comforted» témoigne d'une recherche semblable de la complétude, mais sur le plan métaphysique : le grand héros tragique irlandais, exemple de courage, est accueilli au Paradis par le chœur des lâches auquel il doit se joindre, complétant ainsi sa vie héroïque par la connaissance de son contraire, selon le cycle yeatsien où les antinomies doivent se succéder. "The Black Tower » s'inscrit dans la même dynamique, et, en tant 
que dernier poème, rejaillit sur l'ensemble de l'œuvre poétique de Yeats, qu'un critique comme Hazard Adams ${ }^{6}$ considère au demeurant comme un seul livre de poésie.

Say that the men of the old black tower

Though they but feed as the goatherd feeds

Their money spent, their wine gone sour,

Lack nothing that a soldier needs,

That all are oath-bound men

Those banners come not in.

There in the tomb stand the dead upright

But winds come up from the shore

They shake when the winds roar

Old bones upon the mountain shake.

Those banners come to bribe or threaten

Or whisper that a man's a fool

Who when his own right king's forgotten

Cares what king sets up his rule.

If he died long ago

Why do you dread us so?

There in the tomb drops the faint moonlight

But wind comes up from the shore

They shake when the winds roar

old bones upon the mountain shake.

The tower's old cook that must climb and clamber

Catching small birds in the dew of the morn

When we hale men lie stretched in slumber

Swears that he hears the king's great horn.

But he's a lying hound;

Stand we on guard oath-bound.

There in the tomb the dark grows blacker

But wind comes up from the shore

They shake when the winds roar

old bones upon the mountain shake.

2 De nombreux critiques ont exprimé leur opinion concernant ce poème et, en schématisant, on peut les ranger en deux catégories, ceux qui y voient un poème sombre, négatif et pessimiste, fondant leur argumentation sur la présence, rare chez Yeats, du macabre (les ossements qui claquent au vent), et ceux qui voient une issue positive dans la promesse d'un renouveau après la nuit, d'une renaissance après la mort. Citons Brian Arkins comme représentant la première catégorie: "Yeats's view here is extremely pessimistic, for on earth there is no renewal in sight and in the grave of the poem, where the moonlight of subjectivity was initially "faint", at the end "the dark grows blacker" ". "

3 John Unterecker, quant à lui, s'appuie sur le fait que chez Yeats la fin d'un cycle en inaugure un nouveau, pour défendre une thèse plus optimiste :

Like Yeats in being defenders of a defeated aristocratic order, [the men of the old black tower] necessarily stand on guard against the bribes, threats and whispers of those who carry the banners of corrupt popular politics. Prophetic, the poem brings us closer and 
closer to the end of the lunar cycle. In the second chorus one thin crescent of moon sheds "faint moonlight" into the tomb, but by the last chorus the tomb has got blacker, dark of the moon is about to arrive, and with it the cataclysmic end of an era which will hurl the old bones triumphantly back into the world. The old cook of the last stanza is, of course, a "lying hound" when he swears that the "king's great horn" is already sounding; for that horn cannot sound till the moon blacks out completely and the elemental transformation takes place ${ }^{8}$.

$4 \quad$ Nous reprendrons ce dernier point de vue en nous fondant sur les repères et l'orientation que donnent les autres poèmes du recueil auxquels « The Black Tower » est étroitement relié, et aussi sur la présence prégnante du symbolisme alchimique dans cet ultime poème, présence qui, à notre connaissance, n'a pas été commentée, ni même semble-t-il perçue, par la critique.

Le contenu manifeste du poème (pour reprendre le vocabulaire que Freud applique aux rêves) se traduit clairement dans sa prosodie. La première strophe fait apparaître l'opposition entre la tour et les bannières des assaillants : au martèlement spondaïque de « old black tower » qui ralentit la lecture et rend la tour incontournable répondent avec la même force les trois accents « come not in » qui marquent la forme emphatique. Selon le même schéma rythmique on met en exergue l'adversité "wine gone sour » et aussi la détermination qui lui fait face, celle d'hommes liés par un serment: "oath-bound men ». Les forces conflictuelles sont pour ainsi dire rangées en ordre de bataille sur la page, cet ultime poème développant pour une dernière fois les tensions entre des forces antagonistes, source de création on le sait chez Yeats qui déclarait «all creation is from

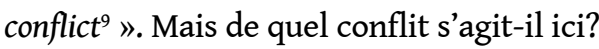

6 L'interprétation de John Unterecker est juste, mais restrictive : il s'agirait des valeurs aristocratiques chères à Yeats face à la politique populiste corrompue. La deuxième strophe apporte quelques éléments : il y est question de corruption, de menaces, d'un roi légitime mort il y a longtemps et d'un pays auquel on impose un nouveau règne. On pense à la lutte de l'Irlande contre l'Angleterre (la dimension héroïque irlandaise est présente à travers les guerriers enterrés debout, d'autre part le poème précédent «Three Songs to the One Burden » fait l'apologie des héros de Pâques 1916), et aussi, dans le contexte politique de janvier 1939, aux défilés, toutes bannières déployées, du nazisme montant qui menace la paix mondiale. Le poème peut aussi faire référence au retour du roi Arthur, ce que corrobore la troisième strophe où le cuisinier entend sonner le cor du roi. Norman Jeffares ${ }^{10}$ rappelle la légende selon laquelle Arthur, Guenièvre, sa cour et une meute de chiens (le mot "hound» figure aussi dans cette strophe) sont endormis dans un caveau sous le château de Sewingshield dans le Northumberland, attendant que quelqu'un sonne du cor, lequel a été abandonné à côté sur une table. Cette piste est donc à privilégier. Les hommes liés par un serment seraient dans cette perspective les chevaliers d'Arthur ${ }^{11}$. Cette interprétation ne contredit nullement celle de Unterecker, le retour du roi Arthur signifiant celui des valeurs traditionnelles, celles du poète. Or la variation des pronoms personnels indique que le poète est de plus en plus impliqué à mesure que le poème se déroule: les défenseurs de la tour sont d'abord «they » dans la première strophe, mais dès la deuxième le poète fait partie des combattants : 
If he died long ago

Why do you dread us so?

西

ultime poème le symbole de la tour dont il a fait un symbole personnel : "I declare this rénovée, dans laquelle il a vécu, et qui a été aussi utilisée jadis par des soldats - « rough men-at-arms $s^{13}$ »- qui ont monté le même escalier. L'adjectif « old» dans « old black tower » renforce l'intimité entre le poète et sa tour. Yeats méditait sur ce symbole et l'utilisait depuis longtemps. En tant que membre de la Golden Dawn, il connaissait l'arcane XVI du Tarot qui était employé dans les rituels de cette société et qui présente une tour frappée par la foudre et un homme qui tombe dans le vide. Or la foudre chez Yeats symbolise l'inspiration poétique ${ }^{14}$. La chute dans le poème se matérialise par son aboutissement, la tombe, présente dans le refrain. Verticalité et horizontalité se répondent dans le poème : la tour verticale, les hommes enterrés debout, les dormeurs allongés ("while we hale men lie stretched in slumber »). Une autre signification de la tour chez les Rose-Croix lui était familière, celle du fourneau hermétique, l'athanor, représenté par une tour. Pour parler de sa vie et de son œuvre Yeats s'exprimait spontanément en termes alchimiques. Ainsi écrivait-il en 1906 : «I am a little disappointed with the upshot of so many years, but I know that I have been busy with the Great Work, no lesser thing than that although it may be the Athanor has burned too fiercely, or too faintly and fitfully, or that the prima materia has been ill-chosen ${ }^{15}$. » Yeats est resté membre de la Golden Dawn de 1890 à 1922 et il était pétri de pensée hermétique. Il n'est donc pas étonnant de retrouver ce vocabulaire dans ce dernier poème où se pose à nouveau la question de l'accomplissement du Grand CEuvre et d'un devenir possible dans l'au-delà.

Le registre alchimique suggère une perspective de renouveau. La tombe ne s'oppose pas à la tour, car en alchimie l'athanor est aussi dénommé «tombeau » pour signifier que la matière première, suppliciée dans le fourneau hermétique, doit d'abord mourir avant de renaître. La descente dans la tombe ouvre ici sur une renaissance. Yeats emploie plusieurs fois la tombe dans ce sens : ainsi sur la couverture de The Secret Rose, un rosier puise ses racines dans une tombe où gît un cadavre vêtu d'une armure, image très proche de celle de «The Black Tower ». Le poème «Broken Dreams» (1915) présente la même perspective : " but in the grave all shall be renewed ${ }^{16}$ »; et " Under Ben Bulben », terminé en septembre 1938 et placé par Yeats au début du recueil des Last Poems deux rangs avant "The Black Tower » dans la table des matières qu'il rédigea lui-même quelques jours avant sa mort, voit dans la mise au tombeau le passage de l'âme vers l'Anima Mundi où elle attend d'être réincarnée :

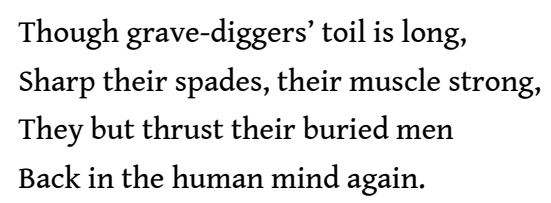

10 La présence de la montagne confirme le lien avec les autres poèmes rosicruciens de Yeats, notamment «The Mountain Tomb» (Responsibilities) où l'on attend le réveil du père Rosencreutz dans son caveau sous la montagne, de même que dans "The Black Tower » on attend le réveil du roi. Cette montagne est la montagne d'Abiegnos chère aux initiés de 
la Golden Dawn : "It is the Mountain of God in the Centre of the Universe, the Sacred Rosicrucian Mountain of Initiation ${ }^{17}$. » A. E. Waite, adepte de la Golden Dawn, et que Yeats connaissait bien, décrit cette montagne dans sa Real History of the Rosicrucians en des termes qui rappellent le poème " The Black Tower » : «When you have discovered this mountain, the first miracle that will appear is this - a most vehement and very great wind will shake the whole mountain and shatter the rocks to pieces ${ }^{18}$.» On reconnaît ici le vent qui rugit sur la montagne et fait claquer les os des squelettes. Il s'agit d'une épreuve initiatique à l'issue de laquelle l'obscurité doit être dissoute: "After all these things and near daybreak, there shall be a great calm, and you shall see the day-star arise, and the darkness will disappear ${ }^{19}$. " Le résultat sera, dans le texte de Waite, la production de l'or. Ce résultat final n'est pas mentionné dans le poème. Mais on peut penser que l'obscurité, la nuit du poème, n'est qu'une étape, un passage débouchant sur la lumière. Le texte met en effet l'accent sur le processus de transformation. Le refrain est identique tout au long du poème, sauf en son premier vers qui varie chaque fois, introduisant la lune, maîtresse du changement, puis mettant l'accent sur le processus évolutif ("There in the tomb the dark grows blacker») en associant le verbe « to grow » au comparatif « blacker».

Le vent, qui semble donner vie aux ossements - les termes «winds » et "shake » se succédant en alternance dans le refrain comme pour mieux l'animer - est le vent du changement que Yeats a pour habitude d'associer aux Sidhe, les divinités irlandaises qui voyagent dans le vent tourbillonnant :

Sidhe is also Gaelic for wind, and certainly the Sidhe have much to do with the wind. They journey in whirling wind, the winds that were called the dance of the daughters of Herodias in the Middle Ages, Herodias doubtless taking the place of some old goddess. When old country people see the leaves whirling on the road they bless themselves, because they believe the Sidhe to be passing by ${ }^{20}$.

Il convient de retenir cette valeur du vent dans un poème où l'Irlande héroïque est éminemment présente. Dans le système exposé dans A Vision, le changement, la succession des périodes historiques mais aussi le passage de la vie à la mort, puis de la mort à une autre vie, est figuré par le double cône ou double spirale, la fin d'un cycle entraînant le début d'un nouveau, la spirale qui se rétrécit donnant naissance à celle qui s'élargit. Dans la poésie, la spirale est évoquée le plus souvent par l'escalier tournant de la tour, lequel n'est pas mentionné dans «The Black Tower ». Stallworthy, dans son étude des brouillons préparatoires des poèmes, nous apprend que la première version commençait ainsi : "I speak for the gyres of the black tower ${ }^{21}$. " Le projet initial comportait donc bien une dimension historique, le pluriel « gyres » suggérant le passage d'une spirale à une autre. Sur le plan historique le poète semble attendre le retour des valeurs de l'Irlande héroïque, et sur le plan personnel, celui de l'homme qui va mourir, une renaissance. Dans la version définitive du poème, le renouveau est annoncé de façon plus discrète car les mots « gyres » et « $I$ » ont été gommés. Où le « $I$ » se situe-t-il ?

La progression du «they " au «we " situe le «I » au-delà du poème. "The Black Tower » entretient des relations de proximité et de similitude avec «Under Ben Bulben », placé deux poèmes avant lui et qui exploite le même thème de la tombe et de la montagne. Dans «Under Ben Bulben » le poète parle d'outre-tombe: "Under bare Ben Bulben's head/In Drumcliff churchyard Yeats is laid [...]» 

cuisinier qui apparaît dans la troisième strophe, la plus manifestement alchimique du poème. Le texte insiste, par le biais de l'allitération (« climb and clamber ») sur l'ascension et sa difficulté. Or, dans les poèmes de la tour, c'est le poète qui effectue cette ascension avant d'arpenter le sommet crénelé et de contempler la campagne environnante (poème " The Tower »). Dans ce contexte ascension signifie spiritualisation et sortie de la spirale du temps vers une autre dimension, la tour, au sommet en ruines, étant décrite comme " half dead at the to ${ }^{23}$ ». Ici le cuisinier, dans le registre alchimique qui se déploie tout au long du poème, n'est autre que l'alchimiste qui entretient le feu de la tour, c'est-à-dire de l'athanor, si bien qu'alchimiste et poète ${ }^{24}$ sont liés. On reconnaît dans cette strophe l'iconographie hermétique traditionnelle : la matière première sous la forme de la rosée (la planche IV du Mutus Liber, manuscrit alchimique du XviII ${ }^{e}$ siècle ${ }^{25}$, présente un couple d'alchimistes récoltant la rosée), l'émanation de vapeurs due à l'exposition de la matière première au feu alchimique étant, selon la tradition, représentée par des oiseaux, cette dernière signification se trouvant renforcée par l'ascension de la tour. Il existe ainsi tout un mouvement symbolique tendant vers la sublimation, la libération. Comment interpréter la défiance des défenseurs de la tour qui ne croient pas le cuisinier dès lors qu'il entend sonner le grand cor du roi ? Le cuisinier-alchimiste, ou poète, en gravissant la tour déploie les spires de l'histoire et accède à une période plus avancée. Comme beaucoup de prophètes il n'est pas compris de ses contemporains. dans ce contexte précis. La couleur noire de la tour n'a pas la valeur de deuil qu'on lui prête dans le christianisme. Elle constitue la première étape réussie dans la réalisation du Grand CEuvre et elle indique que la coction, qui devra ensuite passer par le blanc et le rouge, est en bonne voie. Ce stade de l'œuvre (nigredo) porte aussi parfois le nom de «putréfaction », d'où l'importance des cadavres dans le poème, et Yeats y fait plusieurs fois référence. Ainsi, dans « Rosa Alchemica » : « Our master Avicenna has written that all life proceeds out of corruption ${ }^{26}$.» La matière première doit dans un premier temps être dissoute et retourner au chaos originel avant toute nouvelle recomposition, ce qu'illustre la formule «solve et coagula ». Yeats est fidèle à ce principe dans «Rosa Alchemica » où l'atmosphère s'obscurcit avant toute métamorphose: «I had to struggle again with the shadow, as of some older night than the night of the sun, which began to dim the lights of the candles $[. . .]^{27}$. »

18 La tombe et la tour noire ont donc plutôt le rôle positif de matrice poétique qui donne naissance au poème suivant «Cuchulain Comforted » qui nous entraîne dans un paradis étrange. Pour Jung l'alchimie apportait une compensation nécessaire à la vision chrétienne plus étroite où le bien et le mal sont nettement séparés. L'alchimie voit un ensemble plus complexe basé sur un processus de transformations. Ainsi la matière première corrompue - ici les cadavres - peut-elle être portée par les soins de l'alchimiste à la perfection de la pierre philosophale qui produira l'or incorruptible. Il convient de lire 
« The Black Tower » à la lumière de cette idéologie plus optimiste car visant la perfection et la renaissance.

Le poème développe une tension entre un symbolisme hermétique objectif qui tend vers un renouveau, un devenir dans l'au-delà, et un moi fuyant, éclaté, dont la compréhension de ce phénomène est nécessairement fragmentée. La réalité supérieure unifiée mise en œuvre par le symbolisme alchimique est saisie par un moi diffracté en diverses personae selon un principe moderniste car on ne sait où se situe le poète : s'inclut-il dans le « we » de « stand we on guard oath-bound " ? Ou fait-il simplement parler les défenseurs de la tour au style direct? Se situe-t-il dans l'au-delà comme dans « Under Ben Bulben » ? Ou se faitil représenter par le cuisinier-alchimiste? Il semble que le "je » se décompose par un effet de miroirs propre à l'état de conscience limité et inconstant (discontinu et nonlinéaire) du moi incarné alors que la réalité symbolique de l'Anima Mundi se manifeste avec conviction dans son unité. La réalité ultime, homogène et circonscrite (l'inconscient, ou chez Yeats, la sphère) est perçue par un moi insaisissable. Le « je » absent du poème s'est effacé devant le symbole dont il laisse opérer la force. Ce poème manifeste, une dernière fois, la tension entre Yeats symboliste et Yeats moderniste et démontre ce que le poète, au lendemain de la composition de ce poème, exprimait dans sa correspondance par la formule "man can embody truth but he cannot know it $t^{28}$ ». Le poème en tant qu'acte de langage donne corps à la vérité, mais le moi limité de l'auteur ou du lecteur ne la comprend que partiellement.

\section{NOTES}

1. Brenda Maddox, George's Ghosts, A New Life of W. B. Yeats, London, Basingstoke and Oxford, Picador, 1999, p. 362.

2. The Variorum Edition of the Poems of W. B. Yeats, eds. Peter Allt and Russel K. Alspach, New York, Macmillan, 1977, p. 778.

3. Ibid., p. Xv.

4. Ibid., p. 632.

5. Ibid., p. 639.

6. Hazard Adams, The Book of Yeats's Poems, Tallahassee, The Florida State University Press, 1990.

7. Brian Arkins, Builders of my Soul: Greek and Roman Themes in Yeats, Gerrards Cross, Buckinghamshire, Colin Smythe, 1990, p. 67.

8. John Unterecker, A Reader's Guide to William Butler Yeats, New York, Noonday Press, 1959, p. 292.

9. Autobiographies, London and Basingstoke, Macmillan, 1955, p. 183.

10. A. Norman Jeffares, A Commentary on the Collected Poems of W.B. Yeats, London and Basingstoke, Macmillan, 1968, p. 514. 
11. Roy Foster (W. B. Yeats, A Life, II. The Arch-Poet, New York, Oxford University Press, 2003, p. 648) soutient que le poème s'inspire de la pièce de O'Grady, The Masque of Finn, que Yeats aurait vue trente années auparavant. Dans cette pièce, les compagnons de Finn qui ont survécu attendent le retour de leur roi. Notons que le symbolisme de cette légende est fortement apparenté à celui du récit arthurien.

12. «Blood and the Moon ", The Variorum Edition of the Poems of W. B. Yeats, op. cit., p. 480.

13. « The Tower », ibid., p 412.

14. "[The Daimon's] descending power is neither the winding nor the straight line but zigzag, illuminating the passive and active properties, the tree's two sorts of fruit: it is the sudden lightning, for all his acts of power are instantaneous. We perceive in a pulsation of the artery, and after slowly decline. » (Mythologies, London and Basingstoke, Macmillan, 1959, p. 361).

15. Ibid., p. 849.

16. Ibid., p 356.

17. Israel Regardie, The Golden Dawn, St Paul, MN, Llewellyn, 2002, p. 261.

18. Arthur Edward Waite, Real History of the Rosicrucians, London, 1887, George Redway, p. 444.

19. Ibid.

20. The Collected Poems of W. B. Yeats, edited by Richard J. Finneran, New York, Scribner Paperback Poetry, 1996, p. 454.

21. Jon Stallworthy, Between the Lines, Yeats's Poetry in the Making, London, Oxford University Press, 1963, p. 223.

22. Voir plus haut citation p. 2.

23. « Blood and the Moon", The Variorum Edition of the Poems of W.B. Yeats, op. cit., p. 480.

24. Dans les premières ébauches du poème le cuisinier n'est autre que "Old Tom ", personnage prophétique de "Tom the Lunatic » et « Tom at Cruachan », et autre masque de Yeats. Voir Jon Stallworthy, op. cit., p. 236.

25. Altus Mutus Liber, Reproduction des 15 planches en couleur d'un manuscrit du XVII siècle, introduction et commentaire par Jean Laplace, Milan, Arché, 1979.

26. Mythologies, London and Basingstoke, Macmillan, 1959, p. 276.

27. Ibid., p. 274.

28. The Letters of W.B. Yeats, ed. Allan Wade, London, Rupert Hart-Davis, 1954, p 922.

\section{RÉSUMÉS}

Ce poème, le dernier que Yeats composa, revêt une importance particulière en raison de l'éclairage qu'il est susceptible de porter sur l'ensemble de l'œuvre, que Yeats concevait comme une unité organique, en relation avec son concept d'Unité d'Être. Or ce poème a fait l'objet de lectures divergentes, certains critiques l'interprétant comme une vision pessimiste d'une fin inéluctable alors que d'autres y décèlent une perspective de renouveau. Notre analyse se fonde 
sur la prégnance des symboles de l'art hermétique - la tour, la couleur noire, les cadavres, la tombe, la rosée, les oiseaux - que Yeats connaissait intimement pour les avoir expérimentés au sein de l'Ordre de la Golden Dawn, pour proposer une lecture alchimique qui met l'accent sur le processus de transformation à l'œuvre dans le poème et ses implications métaphysiques.

This poem, the last ever written by Yeats, is of particular relevance as it may shed light on the entire body of Yeats's work, which he considered an organic whole, as shown in his concept of Unity of Being. The different interpretations of the poem are at variance: some critics read it as a pessimistic vision of an inexorable end while others see in it the perspective of a revival. Our analysis is based on the prevalence of the symbols of the Hermetic art - the tower, the black colour, the dead bodies, the tomb, the dew, and the birds - which Yeats knew intimately and had experimented with as a member of the Order of the Golden Dawn. Our interpretation is therefore an alchemical reading highlighting the transformation process at work within the poem and stressing its metaphysical implications.

INDEX

Mots-clés : Yeats W. B., poésie, symbolisme

Keywords : poetry, symbolism, Yeats W. B.

\section{AUTEUR}

\section{MICHEL DUFOUR}

Laboratoire Babel, Université du Sud Toulon-Var 